\title{
Three novel $F 8$ mutations in sporadic haemophilia A cases
}

\author{
Rashid Hussain ${ }^{1 *}$, Noman Bin Abid ${ }^{2}$, Sajjad Hussain ${ }^{3}$, Zeeshan Shaukat ${ }^{3}$, Mudassir Altaf $^{3}$, Sara Altaf $f^{3}$ and \\ Gulzar Niazi ${ }^{3}$
}

Hemophilia A (HA) is an X-linked hereditary disorder characterized by bleeding of variable severity through mild, moderate to severe owing to large range of mutations in the Factor VIII (F8) gene (Bowen 2002). All kind of $F 8$ mutations, except repeats, have been reported for $\mathrm{HA}$, in total up to 2370 (Human Genome Mutation Database 2005). A preliminary study was conducted in our lab for identification of mutations in $F 8$ gene in Pakistani HA patients. Correlation of $F 8$ mutations with clinical manifestation of HA patients was the main objective of the study. Blood samples were collected from $62 \mathrm{HA}$ patients from all over the Pakistan and clinical history of all HA patients was recorded (only patients frequently visiting medical centers for the replacement of Factor VIII were selected for the study). Genomic DNA was extracted from whole blood by standard organic procedure. Specific primers (Figure 1) were designed using "Primer3" (http://biotools.umass med.edu/bioapps/primer3_www.cgi) to amplify the coding region of $F 8$ gene; amplified products were sequenced by ABI 310 and ABI 3100 sequencer (Applied Biosystems, Carlsbad, CA, USA). The sequencing results were visualized using "Chromas 2.33" software (Applied Biosystems) and mutations were detected using "BLAST" software available on the NCBI website (http: balst.ncbi.nlm.nih.gov/Blast.cgi). Three novel mutations (1 deletion; 2 point mutations) were detected in four sporadic HA patients, all from different ethnic backgrounds (Table 1). The deletion of $\mathrm{T}$ in exon 7 within the A1 domain represents a frame-shift change disrupting the protein structure and function, which result in severe manifestation of the disease. A missense point mutation in the A3 domain occurs in codon 1907 at nucleotide number 5720, replacing Serine with Isoleucine, and

\footnotetext{
* Correspondence: raashaiduaar@yahoo.com

'National Institute for Genomics and Advance Biotechnology (NIGAB)/ National Agricultural Research Centre (NARC), Park Road, P.O. Box-NIH, Islamabad, Pakistan

Full list of author information is available at the end of the article
}

confers a moderate type of severity. It should be noted that Serine is a polar and acidic amino acid while Isoleucine is a nonpolar and basic amino acid. A nonsense point-mutation was found in two unrelated patients in the C3 domain (exon 26) and was correlated with moderate clinical findings. Beside these mutations, 27 common SNPs were also detected in $F 8$ gene for the studied patients (Table 2). The allelic data and accession numbers of these SNPs were collected from Ensembl Genome Browser (Ensembl 2000). The results of the study will form the basis not only for an enlarged study but also for diagnosis and genetic counseling of classical hemophilia in Pakistan.

\section{Competing interests}

The authors declare that they have no competing interests.

\section{Author's contributions}

$\mathrm{RH}$ managed the project and wrote the paper. NBA, SH, ZS, MA, SA performed experiments. GN designed the project. All authors read and approved the final manuscript.

\section{Author details}

'National Institute for Genomics and Advance Biotechnology (NIGAB)/ National Agricultural Research Centre (NARC), Park Road, P.O. Box-NIH, Islamabad, Pakistan. ${ }^{2}$ Lahore University of Management Sciences, DHA Phase III Hospital Street 29, Lahore 54792, Pakistan. ${ }^{3}$ National Center of Excellence in Molecular Biology, 87-West Canal Bank Road, Thokar Niaz Baig, Lahore 53700, Pakistan.

Received: 17 April 2012 Accepted: 2 July 2012

Published: 30 July 2012

\section{References}

Bowen DJ (2002) Hemophilia A and B: molecular insights. J clin path; mol path 55:1-18.

Human Genome Mutation Database (2005) Institute of Medical Genetics, Cardiff, http://www.hgmd.cf.ac.uk. Accessed 26 May 2012.

Ensembl (2000) European Molecular Biology Laboratory and Welcome Trust Sanger Institute., http://www.ensembl.org. Accessed 10 June.

doi:10.1186/2193-1801-1-10

Cite this article as: Hussain et al: Three novel F8 mutations in sporadic haemophilia A cases. SpringerPlus 2012 1:10.

\section{实}

(c) 2012 Hussain et al.; licensee Springer. This is an Open Access article distributed under the terms of the Creative Commons Attribution License (http://creativecommons.org/licenses/by/2.0), which permits unrestricted use, distribution, and reproduction in any medium, provided the original work is properly cited. 


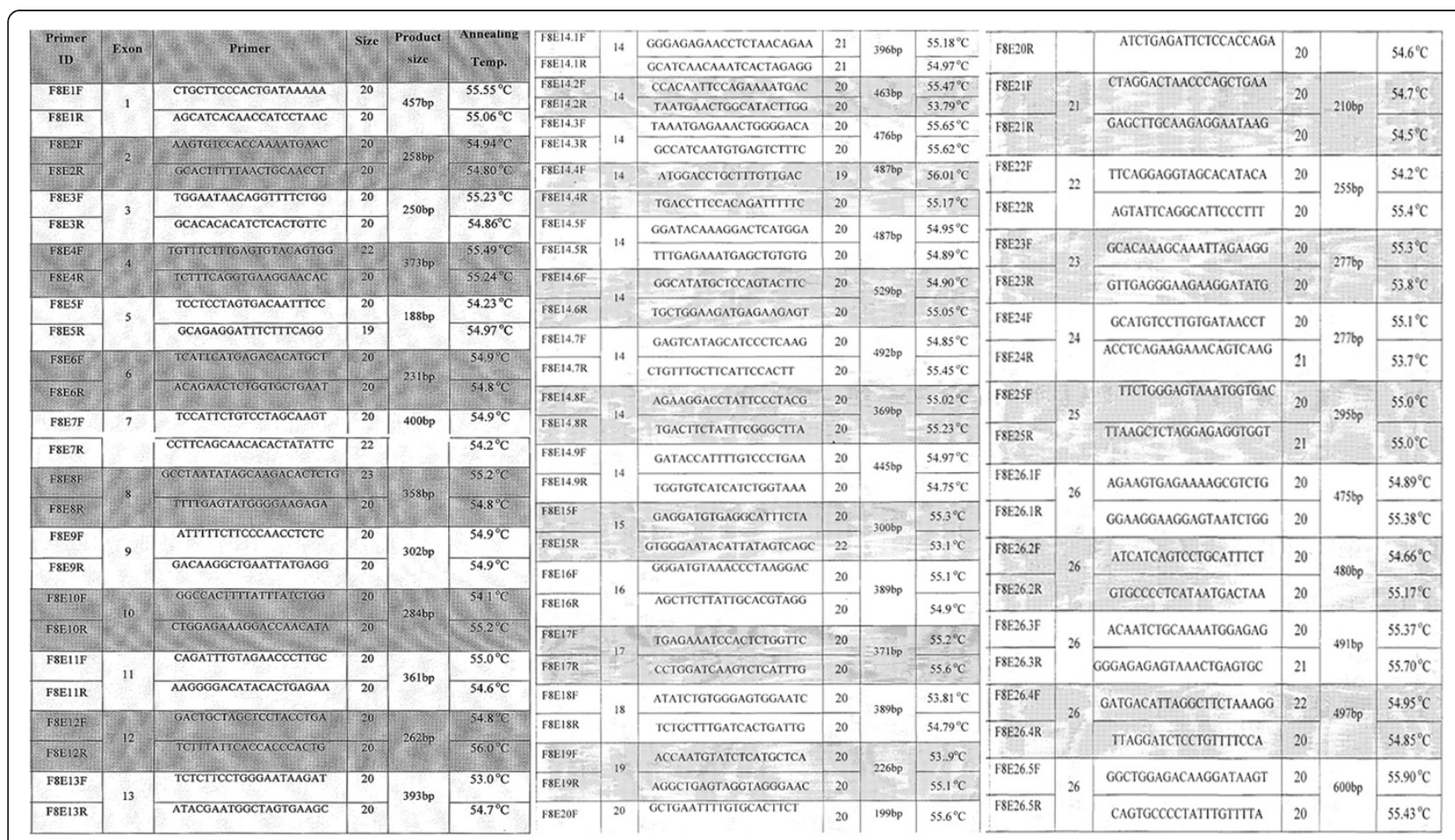

Figure 1 Primers used in the study.

Table 1 Novel mutations in $\mathbf{F 8}$ gene

\begin{tabular}{llllllll}
\hline Age/Sex & Severity & Exon & $\begin{array}{l}\text { Nucleotide } \\
\text { change }\end{array}$ & $\begin{array}{l}\text { Amino acid } \\
\text { change }\end{array}$ & Codon/Codon no. & $\begin{array}{l}\text { Nucleotide genome } \\
\text { ref./cDNA ref. }\end{array}$ & $\begin{array}{l}\text { Affected } \\
\text { Domain }\end{array}$ \\
\hline $4 \mathrm{yr} / \mathrm{male}$ & Severe & 7 & Deletion of T & Frame-shift & CTC $\rightarrow$ C-C/ 318 & $159197688 / 953$ & $\mathrm{~A} 1$ \\
\hline $35 \mathrm{yr} /$ male & Moderate & 17 & $\mathrm{G} \rightarrow \mathrm{T}$ & $\mathrm{Ser} \rightarrow \| \mathrm{le}$ & $\mathrm{AGC} \rightarrow \mathrm{ATC} / 1907$ & $154132724 / 5720$ \\
\hline $15 \& 19 \mathrm{yr} / \mathrm{male}$ & Moderate & 26 & $\mathrm{C} \rightarrow \mathrm{A}$ & $\mathrm{Tyr} \rightarrow$ Termination & TAC $\rightarrow$ TAA 2324 & $154065994 / 6972$ & $\mathrm{~A} 3$ \\
\hline
\end{tabular}

yr (years).

Table $\mathbf{2}$ Common SNPs in $\mathbf{F 8}$ gene (exonic region)

\begin{tabular}{|c|c|c|c|c|c|c|c|c|}
\hline $\begin{array}{l}\text { Sr. } \\
\#\end{array}$ & Patient & Exon & $\begin{array}{l}\text { SNP } \\
\text { ambiguity }\end{array}$ & SNP & Codon & Codon\# & Comments & $\begin{array}{l}\text { Accession } \\
\text { number }\end{array}$ \\
\hline 1 & All 62 Samples & 2 & $W: A / T$ & $\mathrm{~A} / \mathrm{A}$ & GAT & 75 & European $=\mathrm{T} / \mathrm{T}$ & rs1800288 \\
\hline 2 & All 62 Samples & 7 & $\mathrm{~K}: \mathrm{G} / \mathrm{T}$ & $\mathrm{G} / \mathrm{G}$ & TGG & 274 & $\begin{array}{l}\text { European }=C / C ; \\
\text { Spanish Caucasians }=C(0.995) / A(0.005) ; \\
\text { African American, Chinese, Southeast Asia, } \\
\text { Mexican Indian = } C / A\end{array}$ & rs34371500 \\
\hline 3 & All 62 Samples & 8 & $\mathrm{R}: \mathrm{G} / \mathrm{A}$ & $\mathrm{G} / \mathrm{G}$ & CGC & 391 & Ancestral: G & rs137852364 \\
\hline 4 & All 62 Samples & 8 & $Y: T / C$ & $\mathrm{~T} / \mathrm{T}$ & $\underline{T}$ TCA & 392 & European $=\mathrm{C} / \mathrm{C}$ & rs28933669 \\
\hline 5 & All 62 Samples & 8 & $Y: C / T$ & $\mathrm{C} / \mathrm{C}$ & $\underline{T C A}$ & 392 & $?$ & rs28933668 \\
\hline 6 & All 62 Samples & 8 & $\mathrm{~K}: \mathrm{T} / \mathrm{G}$ & $\mathrm{T} / \mathrm{T}$ & ATI & 405 & European $=\mathrm{A} / \mathrm{A}$ & rs28933670 \\
\hline 7 & All 62 Samples & 8 & $\mathrm{R}: \mathrm{A} / \mathrm{G}$ & $\mathrm{A} / \mathrm{A}$ & GAG & 409 & $?$ & rs28933671 \\
\hline 8 & All 62 Samples & 9 & $\mathrm{~K}: \mathrm{G} / \mathrm{T}$ & $\mathrm{T} / \mathrm{T}$ & $\pi \underline{G}$ & 431 & Ancestral: G & rs28933672 \\
\hline 9 & All 62 Samples & 9 & $\mathrm{R}: \mathrm{A} / \mathrm{G}$ & $\mathrm{A} / \mathrm{A}$ & AAA & 444 & Ancestral: G & rs28937272 \\
\hline 10 & All 62 Samples & 9 & $W: T / A$ & $\mathrm{~T} / \mathrm{T}$ & $\underline{T}$ TAC & 450 & Ancestral: A & rs111033616 \\
\hline 11 & All 62 Samples & 10 & $\mathrm{R}: \mathrm{G} / \mathrm{A}$ & $\mathrm{G} / \mathrm{G}$ & CGT & 503 & Ancestral: A & rs35383156 \\
\hline
\end{tabular}


Table 2 Common SNPs in $\mathbf{F 8}$ gene (exonic region) (Continued)

\begin{tabular}{|c|c|c|c|c|c|c|c|c|}
\hline 12 & All 62 Samples & 12 & $Y: T / C$ & $\mathrm{~T} / \mathrm{T}$ & $C T I$ & 622 & Ancestral: $\mathbf{T}$ & rs1800290 \\
\hline 13 & All 62 samples & 15 & $\mathrm{R}: \mathrm{G} / \mathrm{A}$ & $\mathrm{G} / \mathrm{G}$ & CAG & 1764 & Ancestral: A & rs5986891 \\
\hline 14 & All 62 samples & 16 & $R: G / A$ & $\mathrm{G} / \mathrm{G}$ & ATG & 1842 & European = G/G & rs28943674 \\
\hline 15 & All 62 samples & 16 & $Y: C / T$ & $\mathrm{C} / \mathrm{C}$ & $\underline{\mathrm{CCC}}$ & 1844 & European $=\mathrm{C} / \mathrm{C}$ & rs28933675 \\
\hline 16 & All 62 samples & 16 & $\mathrm{M}: \mathrm{A} / \mathrm{C}$ & $\mathrm{A} / \mathrm{A}$ & $\underline{A C T}$ & 1845 & $?$ & rs28933676 \\
\hline 17 & All 62 samples & 16 & $\mathrm{Y}: \mathrm{C} / \mathrm{T}$ & $\mathrm{C} / \mathrm{C}$ & GCC & 1853 & European $=\mathrm{C} / \mathrm{C}$ & rs28933677 \\
\hline 18 & All 62 samples & 17 & $\mathrm{D}: \mathrm{G} / \mathrm{A} / \mathrm{T}$ & $\mathrm{G} / \mathrm{G}$ & GAT & 1865 & Not Available & Cl076951 \\
\hline 19 & All 62 samples & 17 & $\mathrm{R}: \mathrm{A} / \mathrm{G}$ & $\mathrm{A} / \mathrm{A}$ & CAC & 1867 & Ancestral: $\mathbf{G}$ & rs28933679 \\
\hline 20 & All 62 samples & 17 & $\mathrm{~S}: \mathrm{C} / \mathrm{G}$ & $\mathrm{C} / \mathrm{C}$ & C드 & 1873 & European = G/G & rs28933680 \\
\hline 21 & All 62 samples & 17 & $\mathrm{R}: \mathrm{G} / \mathrm{A}$ & $\mathrm{G} / \mathrm{G}$ & $\underline{G A G}$ & 1904 & European $=\mathrm{C} / \mathrm{C}$ & rs28933681 \\
\hline 22 & All 62 samples & 17 & $\mathrm{~S}: \mathrm{G} / \mathrm{C}$ & $\mathrm{G} / \mathrm{G}$ & TGC & 1922 & European = G/G & rs4384155 \\
\hline 23 & All 62 samples & 17 & $\mathrm{~S}: \mathrm{C} / \mathrm{G}$ & $\mathrm{C} / \mathrm{C}$ & TGC & 1922 & European $=\mathrm{C} / \mathrm{C}$ & rs4520342 \\
\hline 24 & All 62 samples & 18 & $\mathrm{R}: \mathrm{A} / \mathrm{G}$ & $\mathrm{A} / \mathrm{A}$ & AAT & 1940 & $?$ & CM083806 \\
\hline 25 & All 62 samples & 18 & $\mathrm{D}: \mathrm{G} / \mathrm{A} / \mathrm{T}$ & $\mathrm{G} / \mathrm{G}$ & CGA & 1960 & $?$ & rs28937294 \\
\hline 26 & All 62 samples & 18 & $\mathrm{R}: \mathrm{G} / \mathrm{A}$ & $\mathrm{G} / \mathrm{G}$ & GGC & 1967 & $?$ & rs111033615 \\
\hline 27 & All 62 samples & 24 & $Y: C / T$ & $\mathrm{C} / \mathrm{C}$ & TAC & 2214 & Ancestral: C & rs1800296 \\
\hline
\end{tabular}

